\title{
Entwicklung und Integration fördern
}

- Laut einer aktuellen Meldung des Statistischen Bundesamtes war 2008 im Durchschnitt jeder siebte Deutsche armutsgefährdet. Vor allem erwerbslose Personen, Menschen mit Behinderungen sowie Alleinerziehende und deren Kinder sind davon betroffen. Doch Armut ist kein deutsches Problem - sie beeinflusst die Gesundheit und das soziale Leben der Betroffenen europaweit. Um das öffentliche Bewusstsein für dieses Thema zu sensibilisieren, hat die Europäische Kommission das Jahr 2010 zum Europäischen Jahr gegen Armut und soziale Ausgrenzung ausgerufen.

_ Öffentlichkeit und Politik sollen mit dem Europäischen Jahr gegen Armut und soziale Ausgrenzung für mehr Engagement gewonnen werden. Auch Physiotherapeuten können bei der Entwicklung von präventiven und gesundheitsfördernden Maßnahmen ihr Know-how zum Besten geben. Vor allem die Themenfelder „Entwicklungschancen bei Kindern verbessern“ und „Integration statt Ausgrenzung - Selbstbestimmte Teilhabe für alle Menschen!“ müssen sie aufgreifen. Denn gesellschaftlich und gesundheitspolitisch Verantwortung zu übernehmen, sollte für eine so große Berufsgruppe selbstverständlich sein.

_ physiopraxis nimmt sich 2010 des Themas an. Studien und Gesundheitsberichte belegen: Die Gehirnfunktion von Kindern und Jugendlichen ist abhängig vom sozioökonomischen Status - bei Kindern aus armen Familien ist sie schlechter. In der Juni-Ausgabe werden wir Ihnen dazu Fakten liefern und aufzeigen, wie Therapeuten hier ihre Fachkompetenz einbringen können.

_ Wie der soziale Status das Leben und die Gesundheit beeinflusst, beschrieb Physiotherapeutin Eva Trompetter bereits in physiopraxis 4/09. Nun hat sie sich mit Kollegen unterhalten, die überwiegend Patienten in sozialen Brennpunkten behandeln. Keine leichte Aufgabe für die Physiotherapeuten. Wie sie dennoch die Situation meistern, fasst die Autorin in der März-Ausgabe für Sie zusammen.

_ Ich wünsche uns allen, dass das Europäische Jahr Erfolge zeigt, alle Kinder gute Entwicklungschancen haben und die Integration gefördert wird.

Herzlichst Ihre

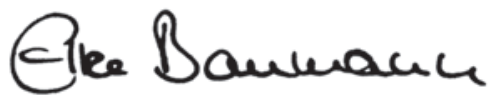

\section{PHYSIOBONUS}

physiobonus heißen die Gewinnspiele in physiopraxis. Wer teilnehmen möchte, nutzt das Formular unter www.thieme.de/ physioonline in der Rubrik physioexklusiv. Und das gibt es in dieser Ausgabe zu gewinnen:

Kursplatz

1 Wochenendlehrgang bei spt-education

Seite 55

Bücher

2-mal „Lehrbuch

Evidenzbasierte Medizin“ Seite 33

3-mal „Handlungsorientiert Lehren und Lernen“"

Seite 38

3-mal „Einführung

in das systemische

Gesundheitscoaching“ Seite 42

und außerdem

1 Stepper

Seite 26 